\title{
Biological Cancer Immunotherapy
}

National Cancer Institute

\section{Source}

National Cancer Institute. Biological Cancer Immunotherapy. NCI Thesaurus. Code C15974.

The use of biological agents to enhance the host immune system in the treatment of cancer. 